Case Report

\title{
A Case of Medication-Resistant Acanthamoeba Keratitis Treated by Corneal Crosslinking in Turkey
}

\author{
Goktug Demirci ${ }^{1}$ and Akif Ozdamar ${ }^{2}$ \\ ${ }^{1}$ Department of Ophthalmology, Medipol University Hospital, 34800 Istanbul, Turkey \\ ${ }^{2}$ Department of Ophthalmology, Cerrahpasa Medical Faculty of Istanbul University, 34800 Istanbul, Turkey
}

Correspondence should be addressed to Goktug Demirci; drgdemirci@yahoo.com

Received 15 November 2013; Accepted 5 December 2013

Academic Editors: K. Jayaraman and G. Kleinmann

Copyright ( 2013 G. Demirci and A. Ozdamar. This is an open access article distributed under the Creative Commons Attribution License, which permits unrestricted use, distribution, and reproduction in any medium, provided the original work is properly cited.

Purpose. To report a case of medication-resistant acanthamoeba keratitis (AK) treated successfully by corneal crosslinking (CXL). Methods. A 26-year-old male with medication-resistant AK underwent a standard CXL procedure with local anesthesia, followed by central corneal epithelial debridement, application of riboflavin $0.1 \%$, and UV-A irradiation. Results. The patient experienced a dramatic symptomatic improvement within 24 hours. At two months, keratitis was healed with a semitransparent paracentral scar that did not affect visual acuity. Conclusions. Our experience, considered in the context of recent studies, suggests that CXL may be an option for selected patients with medication-resistant AK and corneal melting. CXL allows patients to avoid emergency keratoplasty and experience rapid symptomatic relief.

\section{Introduction}

Acanthamoeba keratitis $(\mathrm{AK})$ is a rare, vision-threatening, necrotizing corneal disease caused by small, free-living amoebae belonging to the genus Acanthamoeba. They are typically uninucleated, active, motile trophozoites but form a doublewalled cyst and become dormant when exposed to harsh environmental conditions [1]. The first AK case was reported in 1974 and case reports increased in 1984, particularly among contact lens wearers [2].

AK typically presents with severe pain and associated radial neuritis, inflammation, redness, and photophobia. AK is the most clinically challenging form of ophthalmologic keratitis. In the past, AK symptoms often led ophthalmologists to incorrectly diagnose herpetic keratitis. Mathers et al. showed that, in some series of herpetic keratitis, AK was also present as an opportunistic infection [3]. Combination drug therapy is highly effective only with early diagnosis. With late diagnosis, medical therapy may be insufficient to stop disease progression, and penetrating keratoplasty (PKP) may be required [4].
Japanese scientists demonstrated that, when riboflavin is exposed to visible light or UV light, it can inactivate RNAcontaining tobacco mosaic virus [5]. Recently, several studies have demonstrated the efficacy of UV light in inactivating medication-resistant AK $[6,7]$. Considering this successful application, we performed CXL for the treatment of medication-resistant AK.

\section{Case}

A 26-year-old male with a history of soft contact lens use was referred to our ophthalmology clinic with severe pain, redness, and photophobia in the right eye. Two months ago, he had received topical antibiotics from a general ophthalmologist. Because the corneal ulcer did not heal, he was referred to a corneal specialist. Confocal microscopy and corneal scraping cultures demonstrated acanthamoeba infection. Despite antiacanthamoebal treatment with $1 \mathrm{mg} / \mathrm{mL}$ propamidine isethionate eye drops (Brolene, Aventis Pharma) hourly and chlorhexidine $0.02 \%$ eye drops $(200 \mathrm{mu} / \mathrm{mL})$ hourly, the patient's keratitis worsened. Physical examination revealed a 


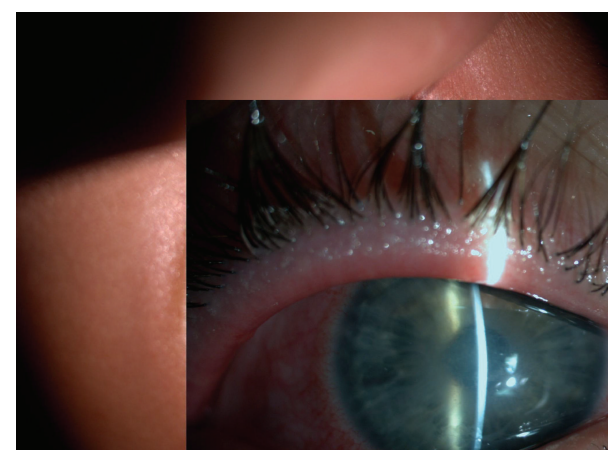

FIGURE 1: Ring ulcer and stromal necrosis of cornea.

ring ulcer, typical of acanthamoeba, with stromal necrosis (Figure 1). Confocal corneal examination revealed doublewalled acanthamoebal cysts throughout the cornea (Figure 2), as well as extreme corneal nerve thickening (Figure 3). Based on our assessment of medication-resistant acanthamoeba keratitis with melting, we performed CXL according to the Dresden protocol and forewent immediate PKP.

CXL treatment for AK keratitis is nearly identical to standard protocol keratoconus treatment. However, they differ in that only loose epithelium and epithelium around the infectious site are removed following anesthetic eye drop application in AK keratitis. Following administration of topical $0.1 \%$ tetracaine every 5 minutes for 15 minutes, we debrided the central $9 \mathrm{~mm}$ of corneal epithelium and acanthamoebal infiltrates. Next, $0.1 \%$ aqueous riboflavin phosphate (vitamin B2) drops were administered every 3 min for $30 \mathrm{~min}$. The eye was then irradiated for 30 minutes with UV-A, at a working distance of $5 \mathrm{~cm}$. Riboflavin was reapplied every 5 minutes during the 30 -minute irradiation period. The patient experienced dramatic symptomatic improvement, especially pain reduction, over 24 hours. Moxifloxacin drops (Vigamox, Alcon) were administered four times daily. All ancillary inflammatory signs resolved after several days. Corneal melting was arrested and complete epithelialization was achieved in 10 days. During the first month of followup, there were no signs of active keratitis by clinical exam or confocal microscopy. At one month, we prescribed dexamethasone $0.1 \%$ eye drops (Dexa-Sine SE, Liba) (Figure 4). After two months, keratitis healed, and the patient had a semitransparent paracentral scar that did not affect visual acuity.

\section{Discussion}

Most patients presenting with $\mathrm{AK}$ are soft contact lens wearers [3]. It is thought that contact lenses create corneal abrasions by microtrauma, which facilitates acanthamoebal entry. Animal studies show that amoebae are pathologic in the context of compromised epithelium $[8,9]$. Tap water rinsing of contact lenses allows deposition of lime scale deposits, which often contain pathogenic Acanthamoeba species [10]. However, some AK patients do not have this

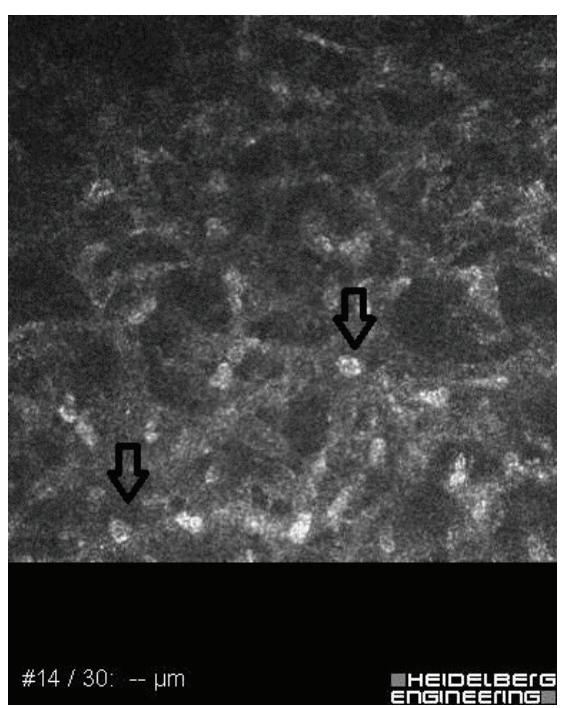

Figure 2: Confocal corneal examination revealing double-walled acanthamoebal cysts.

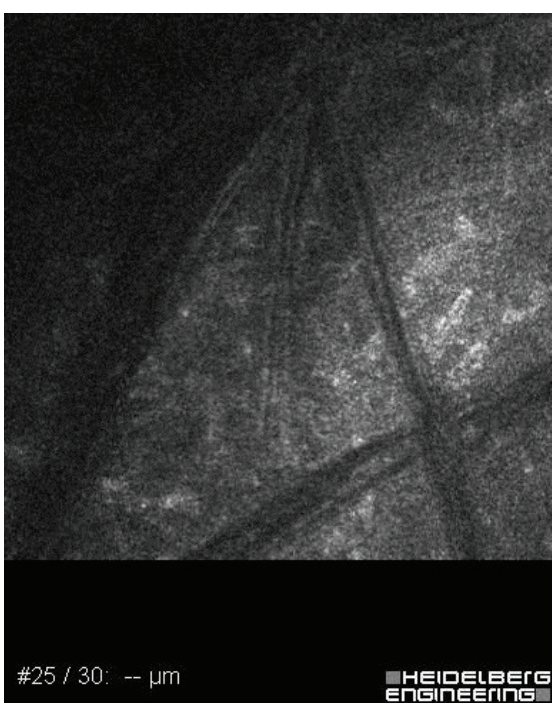

FIGURE 3: Confocal corneal examination revealing extreme corneal nerve thickening.

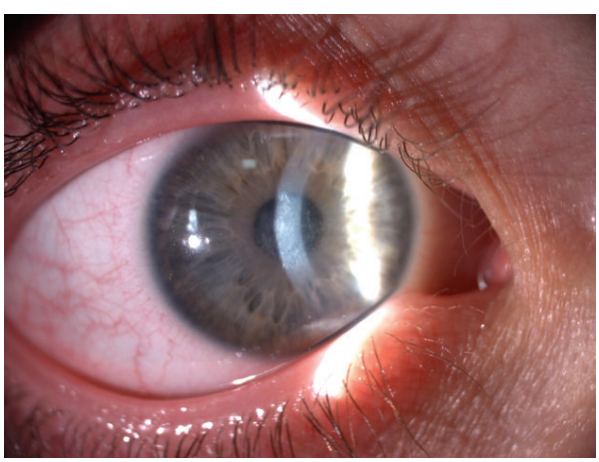

Figure 4: Biomicroscopy of cornea at the end of first month revealing complete resolution of keratitis. 
history, and persons who do not wear contact lenses have also been affected by AK [11].

Combination drug therapy is highly successful when the disease is diagnosed early, but there is no gold-standard treatment. In contrast to bacterial ulcers, AKs do not progress rapidly; therefore, the practitioner has an opportunity to successfully intervene, even if signs and symptoms have been present for one month [9].

In 2008, Iseli et al. described a series of five patients who underwent successful CXL to treat infectious corneal melts which had failed conventional antimicrobial pharmacotherapy. They suggested two possible mechanisms for immediate regression of the corneal melting process [12]. First, CXL may increase collagen resistance to digestive enzymes such as pepsin, trypsin, and collagenase. Approximately $90 \%$ of corneal thickness is stroma, which is composed of regularly arranged collagen fibrils with keratocytes. Bacteria and fungi produce enzymes that digest human collagen and cause corneal melting. Secondly, cell apoptosis following CXL may occur for both human keratocytes and pathogens due to the antimicrobial effect of UV-A light $[12,13]$. Several case reports have documented responses to CXL therapy in complicated infectious keratitis $[6,7,14,15]$. In 2010, Makdoumi et al. performed the first prospective study of CXL as a first-line treatment for bacterial corneal ulcers. Of 16 patients receiving CXL as primary therapy for suspected bacterial keratitis, only two required antibiotic treatment [14]. The antibacterial effectiveness of CXL has been demonstrated in vitro against Staphylococcus aureus, Staphylococcus epidermidis, and Streptococcus pneumoniae grown on agar plates. Martins et al. showed limited effects for Pseudomonas aeruginosa and no measurable effect for Candida albicans [16]. In vitro effects of UV-A and riboflavin on acanthamoebae are less impressive than those observed in vivo effects. However, in vitro results do not always translate to in vivo efficacy $[17,18]$.

Despite these promising results, the above-referenced studies were limited by small case numbers. Further investigations are needed before CXL can be incorporated into routine clinical practice for keratitis treatment.

Our experience of a rapid response with a rapid subjective reduction in pain and photophobia differs from experiences with CXL for keratoconus, in which pain remains until epithelization completion. This difference may be due to the rapid atrophic keratoneuritis response of corneal nerve plexus; it was reported that subepithelial stromal nerve fibers disappeared immediately after CXL and were restored one year later with full corneal sensitivity [19]. Makdoumi et al. have also reported rapid symptomatic relief and believe that this results from ocular immune-response effects, which may be involved in corneal melting through stromal immune-cell modulation [14]. Khan et al. theorize that oxidative processes during CXL may deplete the natural nutritional source to microorganisms, thus acting as a cysticidal agent. In addition, activated flavin and free radical insult to DNA or RNA may damage microorganism genomes. Others believe that the direct damaging effect of UV light is unlikely to produce antimicrobial effects [7].

In consideration of our current understanding of CXL, this rapid response may be due to thermomechanical harm to
AK trophozoites, which would be exposed to temperatures of nearly $75^{\circ} \mathrm{C}$ in the anterior stroma during this procedure [20].

\section{Conclusion}

To our knowledge, this is the first reported case of successful CXL treatment for AK keratitis in Turkey. Our case suggests that CXL may be a promising option in selected cases of medication-resistant AK with corneal melting. CXL treatment may allow patients to avoid emergency keratoplasty and provide rapid symptomatic relief, but further investigations are needed.

\section{Disclosure}

This paper is an original case report. It has not been published anywhere.

\section{Conflict of Interests}

The authors declare that they do not have any financial or proprietary interests in any of the materials.

\section{References}

[1] J. Naginton, P. G. Watson, T. J. Playfair, J. McGill, B. R. Jones, and A. D. Steele, "Amoebic infection of the eye," The Lancet, vol. 2, no. 7896, pp. 1537-1540, 1974.

[2] G. S. Visvesvera, "Classification of Acanthamoeba," Reviews of Infectious Diseases, vol. 13, pp. 369-372, 1991.

[3] W. D. Mathers, M. A. Goldberg, J. E. Sutphin et al., "Coexistent Acanthamoeba keratitis and herpetic keratitis," Archives of Ophthalmology, vol. 115, no. 6, pp. 714-718, 1997.

[4] S. A. Sadiq, A. Azuara-Blanco, D. Bennett, J. H. Lloyd, and H. S. Dua, "Evaluation of contamination of used disposable contact lenses by Acanthamoeba," CLAO Journal, vol. 24, no. 3, pp. 155$158,1998$.

[5] A. Tsugita, Y. Okada, and K. Uehara, "Photosensitized inactivation of ribonucleic acids in the presence of riboflavin," $B B A$ Section Nucleic Acids And Protein Synthesis, vol. 103, no. 2, pp. 360-363, 1965.

[6] K. Makdoumi, J. Mortensen, and S. Crafoord, "Infectious keratitis treated with corneal crosslinking," Cornea, vol. 29, no. 12, pp. 1353-1358, 2010.

[7] Y. A. Khan, R. T. Kashiwabuchi, S. A. Martins et al., "Riboflavin and ultraviolet light a therapy as an adjuvant treatment for medically refractive acanthamoeba keratitis: report of 3 cases," Ophthalmology, vol. 118, no. 2, pp. 324-331, 2011.

[8] F. Van Klink, H. Alizadeh, Y. He et al., "The role of contact lenses, trauma, and Langerhans cells in a Chinese hamster model of Acanthamoeba keratitis," Investigative Ophthalmology and Visual Science, vol. 34, no. 6, pp. 1937-1944, 1993.

[9] J. P. McCulley, H. Alizadeh, and J. Y. Niederkorn, "The diagnosis and management of Acanthamoeba keratitis," CLAO Journal, vol. 26, no. 1, pp. 47-51, 2000.

[10] F. Stapleton, D. V. Seal, and J. Dart, "Possible environmental sources of Acanthamoeba spp in contact lens wearers," British Journal of Ophthalmology, vol. 76, no. 7, pp. 424-427, 1992.

[11] G. Demirci, G. M. Ay, L. V. Karabaş, Ö. Altintaş, G. S. Tamer, and Y. Çağlar, "Acanthamoeba keratitis in a 5-year-old boy without a 
history of contact lens usage," Cornea, vol. 25, no. 3, pp. 356-358, 2006.

[12] H. P. Iseli, M. A. Thiel, F. Hafezi, J. Kampmeier, and T. Seiler, "Ultraviolet a/riboflavin corneal cross-linking for infectious keratitis associated with corneal melts," Cornea, vol. 27, no. 5, pp. 590-594, 2008.

[13] E. Spoerl, G. Wollensak, D. Dittert, and T. Seiler, "Thermomechanical behavior of collagen-cross-linked porcine cornea," Ophthalmologica, vol. 218, no. 2, pp. 136-140, 2004.

[14] K. Makdoumi, J. Mortensen, O. Sorkhabi, B. Malmvall, and S. Crafoord, "UVA-riboflavin photochemical therapy of bacterial keratitis: a pilot study," Graefe's Archive for Clinical and Experimental Ophthalmology, vol. 250, no. 1, pp. 95-102, 2012.

[15] H. Morén, M. Malmsjö, J. Mortensen, and A. Öhrström, "Riboflavin and ultraviolet a collagen crosslinking of the cornea for the treatment of keratitis," Cornea, vol. 29, no. 1, pp. 102-104, 2010.

[16] S. A. R. Martins, J. C. Combs, G. Noguera et al., "Antimicrobial efficacy of riboflavin/UVA combination $(365 \mathrm{~nm})$ in vitro for bacterial and fungal isolates: a potential new treatment for infectious keratitis," Investigative Ophthalmology and Visual Science, vol. 49, no. 8, pp. 3402-3408, 2008.

[17] R. T. Kashiwabuchi, F. R. S. Carvalho, Y. A. Khan et al., "Assessing efficacy of combined riboflavin and UV-A light $(365 \mathrm{~nm})$ treatment of Acanthamoeba trophozoites," Investigative Ophthalmology \& Visual Science, vol. 52, no. 13, pp. 93339338, 2011.

[18] M. A. Del Buey, J. A. Cristóbal, P. Casas et al., "Evaluation of in vitro efficacy of combined riboflavin and ultraviolet A for Acanthamoeba isolates," American Journal of Ophthalmology, vol. 153, no. 3, pp. 399-404, 2012.

[19] C. Mazzotta, C. Traversi, S. Baiocchi, P. Sergio, T. Caporossi, and A. Caporossi, "Conservative treatment of keratoconus by riboflavin-UVA-induced cross-linking of corneal collagen: qualitative investigation of corneal epithelium and subepithelial nerve plexus regeneration by in vivo HRT II system confocal microscopy in humans," European Journal of Ophthalmology, vol. 16, no. 4, pp. 530-535, 2006.

[20] J. L. Griffin, "Temperature tolerance of pathogenic and nonpathogenic free-living amoebas," Science, vol. 178, no. 4063, pp. 869-870, 1972. 


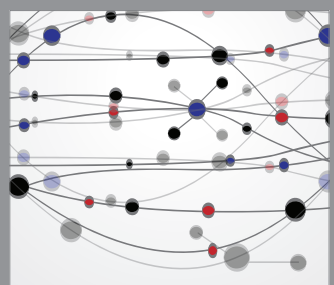

The Scientific World Journal
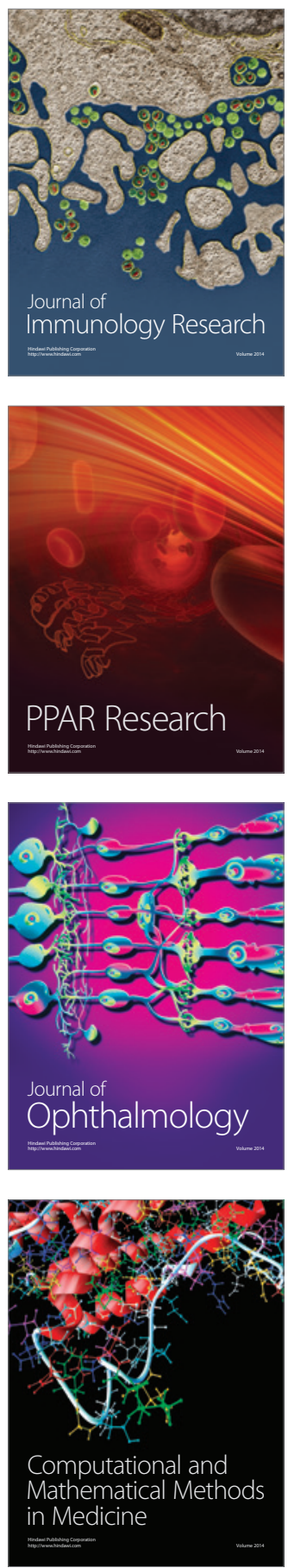

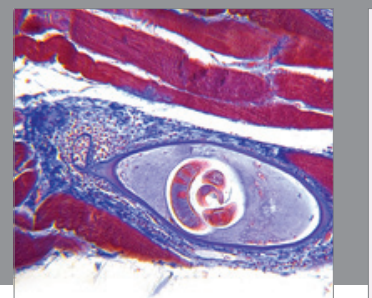

Gastroenterology

Research and Practice
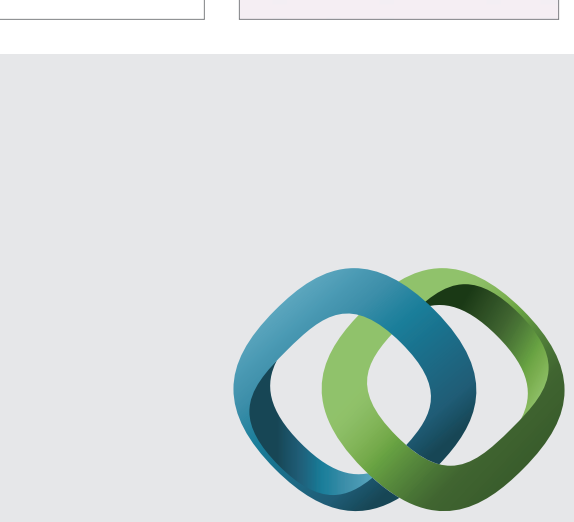

\section{Hindawi}

Submit your manuscripts at

http://www.hindawi.com
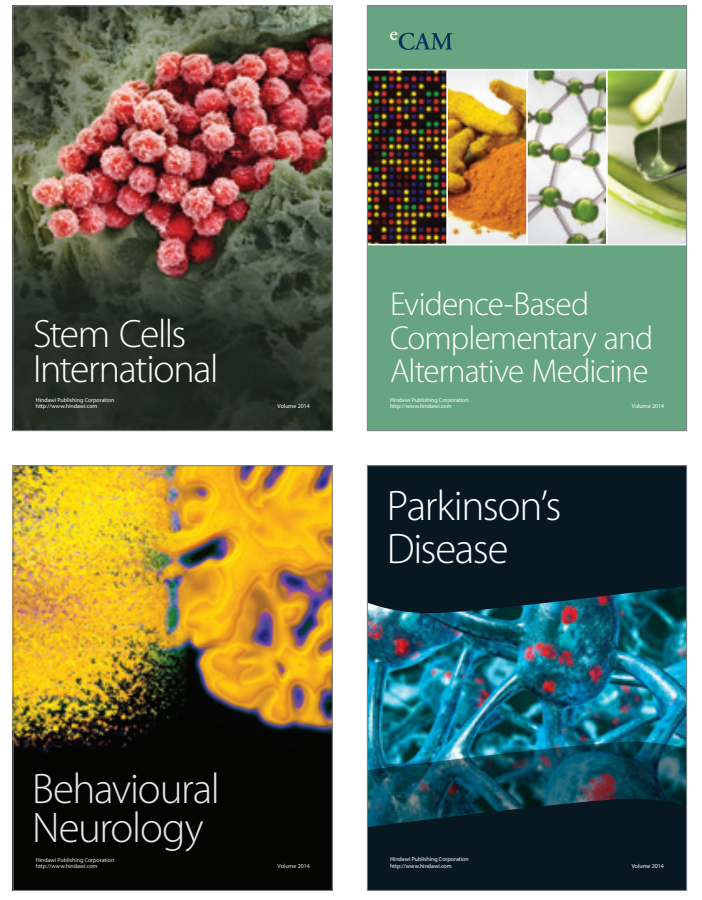
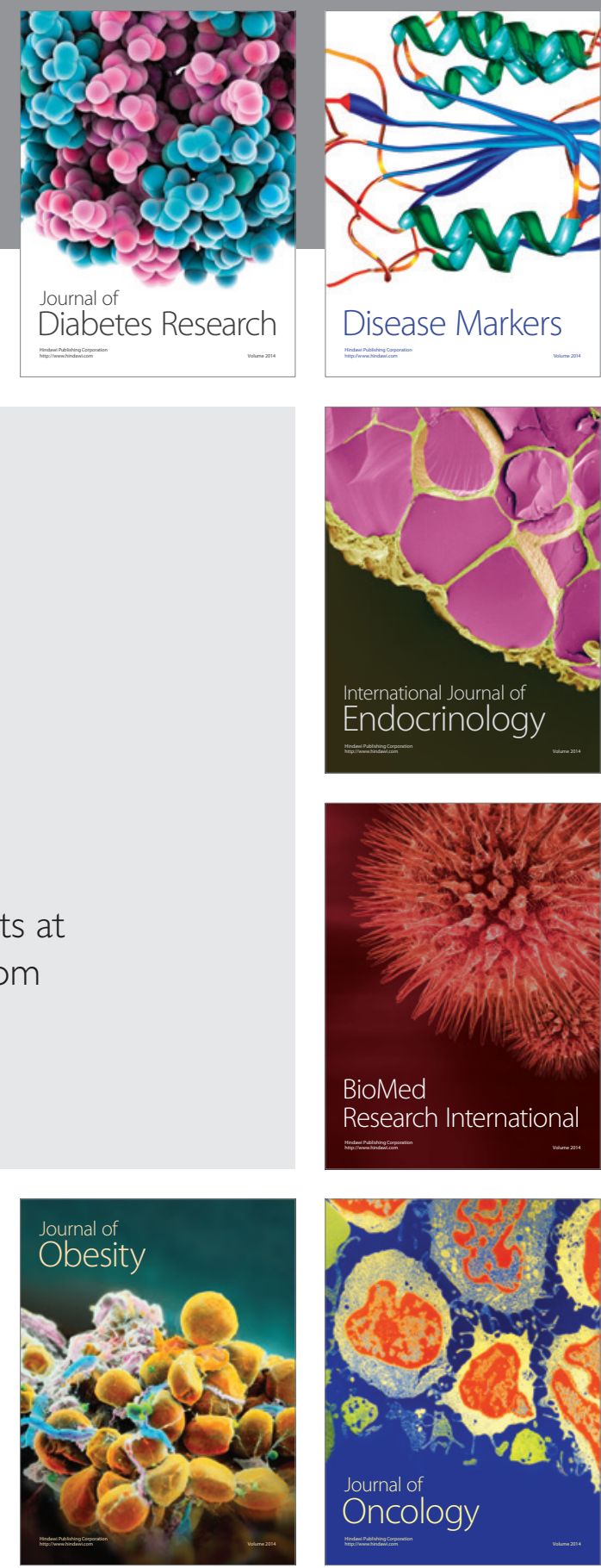

Disease Markers
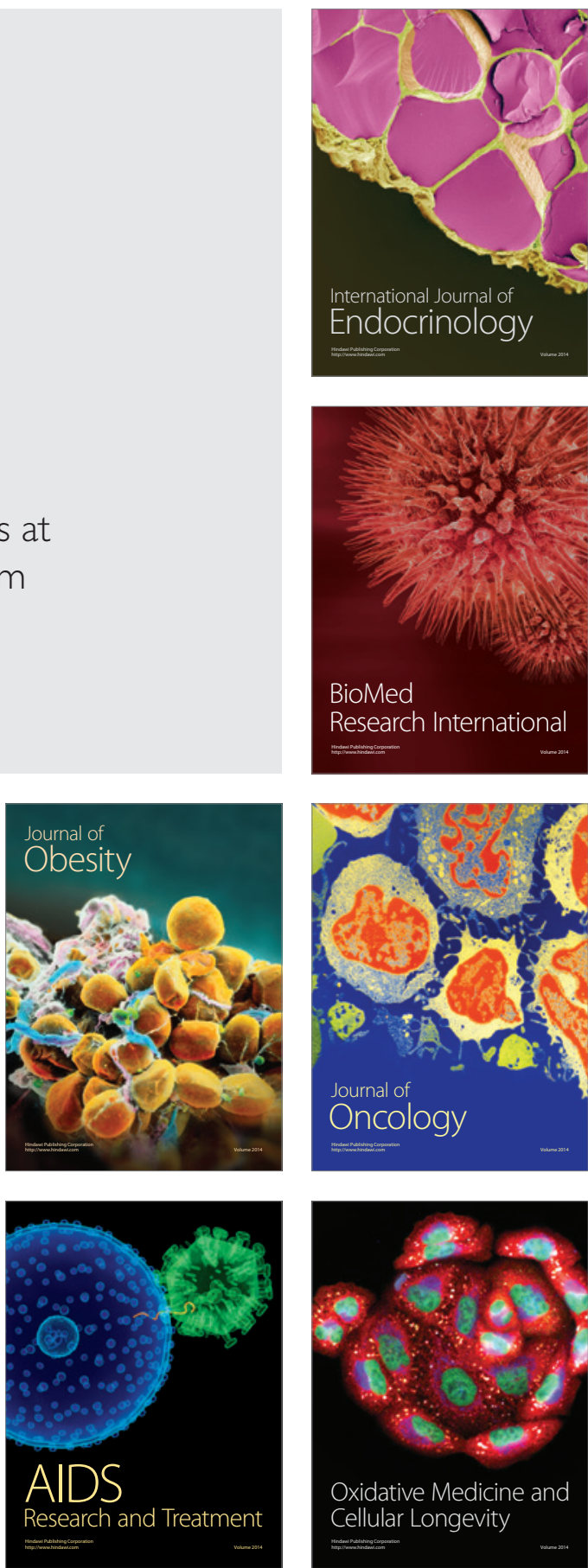